\title{
MULTIPLICATIVE TRANSFERS IN ORDINARY COHOMOLOGY
}

\author{
by RICHARD STEINER
}

(Received 24th March 1980)

\section{Introduction}

In [10] Segal shows that the groups of units in certain ordinary cohomology rings are the zeroth terms of generalised cohomology theories. Geometric methods then give a multiplicative transfer on these groups of units for fibrations with finite fibres; see Kahn and Priddy [6] and Adams ([1], 4). On the other hand Evens [5] by manipulations with cochains has constructed a multiplicative transfer in the cohomology of a group $G$ and a subgroup $H$ of finite index. Now it is well known that the algebraic cohomology of $G$ and $H$ can be identified with the topological cohomology of their classifying spaces $B G$ and $B H$, and that there is a fibration $B H \rightarrow B G$ with finite fibres. This suggests that Evens' algebraic transfer and the geometric transfer derived from Segal's work may be related. In the present paper I confirm this by constructing a common generalisation; I also describe some of its properties.

The construction of this generalised transfer is given in Section 4. It is the construction of the geometric transfer in [1], 4.2, generalised to allow local coefficients. To show that the construction also generalises Evens' transfer it is described in terms of cochains in Section 6 (compare Theorem 6.1 below with 4.5 and 5.5 of [5]). The formal properties of the transfer are stated in Section 3 and proved in Section 5. They correspond to those of the geometric transfer as stated in [1], 4.3, so far as they are relevant. They also imply the formal properties of the algebraic transfer as stated in [5], 6 , except for the double coset rule, which is proved in Section 7. Section 2 contains remarks about the coefficient groups.

When I began this work I hoped that the algebraic transfer would be a special case of the usual geometric transfer, so that the properties of the algebraic transfer could be deduced from those of the geometric transfer without extra work. This fails, partly because of the need to generalise the geometric transfer, partly because we are using the wrong construction of it. The transfer in a cohomology theory for a fibration with finite fibres can be regarded as a construction on the fibration (as in [1],4.1) or on the space representing the cohomology theory (as in [1], 4.2). It is the former construction that has been most studied and the latter that is generalised here. The result of all this is that the work in Section 5 generalises proofs that are well known but have not been published (so far as I know). Even so, I think the proofs in Section 5 are easier than ones involving cochains would be; and the proof of the double coset rule (Section 7), which follows the ideas of [1], end of 4.3, may be illuminating.

I am grateful to Professor J. F. Adams for telling me of Evens' transfer and suggesting comparing it with the geometric transfer. 


\section{Coefficient groups}

Let $p:(X, A) \rightarrow(Y, B)$ be a fibration of $C W$ complexes with finite fibres (we require that $\left.A=p^{-1}(B)\right)$. We have to define multiplicative transfers from cohomology classes of $(X, A)$ to cohomology classes of $(Y, B)$. To see what cohomology classes to take we make three observations.

2.1. Let $G^{*}$ be one of Segal's cohomology theories [10], constructed from a graded ring $R=\left(R_{0}, R_{1}, \ldots\right)$. Then $G^{0}(X)$ is the group of units in

$$
H^{*}(X ; R)=\prod_{q=0}^{\infty} H^{q}\left(X ; R_{q}\right)
$$

So $G^{0}$ is represented by the product of Eilenberg-MacLane spaces

$$
K(R)^{*}=K\left(R_{0}^{*}, 0\right) \times \prod_{q=1}^{\infty} K\left(R_{q}, q\right)
$$

where $R_{0}^{*}$ denotes the group of units in $R_{0}$. We see that the path-components of $K(R)^{*}$ are in bijection with $R_{0}^{*}$, and that the base-point is in the path-component corresponding to 1 . Now $G^{0}(X, A)$ is the set of homotopy classes of maps from $X$ to $K(R)^{*}$ sending $A$ to the base-point; it follows that $G^{0}(X, A)$ is not, as one might expect, a subset of $H^{*}(X, A ; R)=\prod_{q=0}^{\infty} H^{q}\left(X, A ; R_{q}\right)$. However $G^{0}(X, A)$ is a subset of

$$
H^{0}\left(X ; R_{0}\right) \times \prod_{q=1}^{\infty} H^{q}\left(X, A ; R_{q}\right)
$$

2.2. The most general results on the cup-product are obtained from products of the form

$$
H^{*}(; M) \times H^{*}(; N) \rightarrow H^{*}(; M \otimes N)
$$

( $M$ and $N$ abelian groups) rather than of the form

$$
H^{*}(; R) \times H^{*}(; R) \rightarrow H^{*}(; R)
$$

( $R$ a ring). For a similar reason we shall use graded abelian groups in this paper rather than graded rings.

2.3. To obtain Evens' transfer in the generality of [5] one must use local coefficients.

Following 2.2 and 2.3 we shall use as coefficients for the cohomology classes of $(X, A)$ graded local systems on $X$; these are defined to be functors from $\pi X$, the fundamental groupoid of $X$, to the category of graded abelian groups of the form

$$
M=\left(M_{0}, M_{1}, \ldots\right)
$$


Following 2.1 we take as domain of the transfer the somewhat strange-looking cohomology sets

$$
\mathscr{H}^{*}(X, A ; M)=H^{0}\left(X ; M_{0}\right) \times \prod_{q=1}^{\infty} H^{q}\left(X, A ; M_{q}\right) .
$$

The transfer is to be a sort of "cup-product along the fibres" of $p$, so its codomain will be

$$
\mathscr{H}^{*}\left(Y, B ; p_{\otimes} M\right)
$$

where $p_{\otimes} M$ is the graded local system on $Y$ given by

$$
\left(p_{\otimes} M\right)(y)=\bigotimes_{p(x)=y} M(x) \quad \text { for } \quad y \in Y
$$

We use the anticommutative convention for the tensor product of a finite family of graded abelian groups: for two factors we have

$$
(M \otimes N)_{r}=\bigoplus_{p+q=r} M_{p} \otimes N_{q}
$$

and $M \otimes N$ is identified with $N \otimes M$ by

$$
m \otimes n=(-1)^{p q} n \otimes m \quad \text { for } \quad m \in M_{p}, \quad n \in N_{q}
$$

for more than two factors the tensor product is given by induction; the tensor product of the empty family of graded abelian groups is to be

$$
(\mathbb{Z}, 0,0, \ldots)
$$

( $\mathbb{Z}$ denotes the integers) and the tensor product of the empty family of elements is to be $1 \in \mathbb{Z}$.

Remark 2.6. The conventions on empty families are not arbitrary; they are arranged to satisfy the usual universal properties. Indeed

$$
*+1:(\{*\}, \varnothing, \varnothing, \ldots) \rightarrow(\mathbb{Z}, 0,0, \ldots)
$$

is universal among graded 0-linear maps from the product of the empty family of graded abelian groups to a graded abelian group (0-linearity is a vacuous condition).

So our basic transfer will map $\mathscr{H}^{*}(X, A ; M)$ to $\mathscr{H}^{*}\left(Y, B ; p_{\otimes} M\right)$. A morphism of graded local systems over $Y$ from $p_{\otimes} M$ to $N$, say, will therefore give a transfer from $\mathscr{H}^{*}(X, A ; M)$ to $\mathscr{H}^{*}(Y, B ; N)$. The transfer derived from Segal's work [10] maps a subset of $\mathscr{H}^{\star}(X, A ; R)$ to a subset of $\mathscr{H}^{*}(Y, B ; R)$ where $R$ is a constant anticommutative graded ring; this comes from the morphism $p_{\otimes} R \rightarrow R$ given by multiplication in $R$. The transfer of Evens [5]

$$
\prod_{q=0}^{\infty} H^{2 q}(H ; L) \rightarrow \prod_{q=0}^{\infty} H^{2 q}\left(G ; \mathscr{M}_{H \rightarrow G}(L)\right)
$$


( $G$ a group, $H$ a subgroup of finite index, $L$ an $H$-module, $\mathscr{M}_{H \rightarrow G}(L)$ the monomial $G$ module as defined in [5], 3) can be obtained by taking $X=B H, Y=B G, A=B=\varnothing$,

$$
M=(L, 0, L, 0, \ldots) \quad \text { and } \quad N=\left(\mathscr{M}_{H \rightarrow G}(L), 0, \mathscr{M}_{H \rightarrow G}(L), 0, \ldots\right)
$$

(We remark that graded $H$-modules and graded local systems on $B H$ are equivalent, since $H$ and $\pi B H$ are equivalent groupoids, and similarly for $G$.) To describe the morphism from $p_{\otimes} M$ to $N$ we note that $\left(p_{\otimes} M\right)_{q}$ is zero if $q$ is odd and a direct sum of copies of $\mathscr{M}_{H \rightarrow G}(L)$ if $q$ is even; the morphism is obtained by identifying copies.

We next mention two obvious constructions on graded local systems. First, if $M$ is a graded local system on $X$ and

$$
f:\left(X^{\prime}, A^{\prime}\right) \rightarrow(X, A)
$$

is a map of pairs, then as in the ungraded case we have an induced graded local system $f^{*} M$ on $X^{\prime}$ and an induced map

$$
f^{*}: \mathscr{H}^{*}(X, A ; M) \rightarrow \mathscr{H}^{*}\left(X^{\prime}, A^{\prime} ; f^{*} M\right) .
$$

Second, if $M$ and $N$ are graded local systems on $X$, then they have a tensor product $M \otimes N$, namely the graded local system on $X$ given by

$$
(M \otimes N)(x)=M(x) \otimes N(x) \quad \text { for } \quad x \in X,
$$

and there is a cup-product

$$
\mathscr{H}^{*}(X, A ; M) \times \mathscr{H}^{*}(X, A ; N) \rightarrow \mathscr{H}^{*}(X, A ; M \otimes N)
$$

constructed as in Steenrod [13], 11.

Now suppose that $M$ is a graded local system on $X$. Then the map $p_{\otimes}$ from graded local systems on $X$ to graded local systems on $Y$ has the following obvious properties.

2.7. The $\operatorname{map} p_{\otimes}$ is a functor.

2.8. If

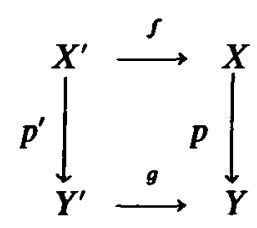

is a pull-back, then $p_{\otimes}^{\prime} f^{*} M$ is naturally equivalent to $g^{*} p_{\otimes} M$.

2.9. If $p$ is the identity, then $p_{\otimes} M=M$.

2.10. If $q: Y \rightarrow Z$ is also a fibration with finite fibres, then $q_{\otimes} p_{\otimes} M$ is naturally equivalent to $(q p)_{\otimes} M$. 
2.11. If $X$ is empty and $M$ is the unique graded local system on $X$, then $p_{\otimes} M$ is the constant graded local system with

$$
\left(p_{\otimes} M\right)(y)=(\mathbb{Z}, 0,0, \ldots) \quad \text { for } \quad y \in Y
$$

2.12. If $X$ is a disjoint union $X_{1} \sqcup X_{2}$ with inclusions

$$
i_{1}: X_{1} \rightarrow X, \quad i_{2}: X_{2} \rightarrow X
$$

then $p_{\otimes} M$ is naturally equivalent to

$$
\left(p i_{1}\right)_{\otimes} i_{1}^{*} M \otimes\left(p i_{2}\right)_{\otimes} i_{2}^{*} M
$$

2.13. If $N$ is also a graded local system on $X$, then $p_{\otimes}(M \otimes N)$ is naturally equivalent to $p_{\otimes} M \otimes p_{\otimes} N$.

\section{The formal properties of the transfer}

Let $p:(X, A) \rightarrow(Y, B)$ be a fibration of $C W$ complexes with finite fibres and let $M$ be a graded local system on $X$. In Section 4 we shall construct a transfer map (not a homomorphism)

$$
p_{\cup}: \mathscr{H}^{*}(X, A ; M) \rightarrow \mathscr{H}^{*}\left(Y, B ; p_{\otimes} M\right)
$$

(the domain and codomain are defined in Section 2) with the following properties (to be proved in Section 5).

3.1. If $\alpha: M \rightarrow N$ is a morphism of graded local systems on $X$, which induces $\alpha: p_{\otimes} M \rightarrow p_{\otimes} N$ since $p_{\otimes}$ is a functor (2.7), then the diagram

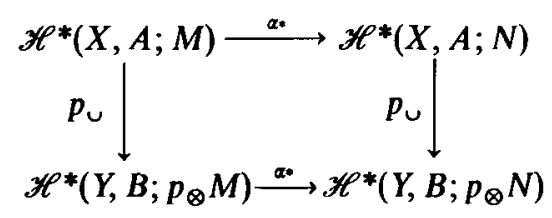

commutes.

3.2. If

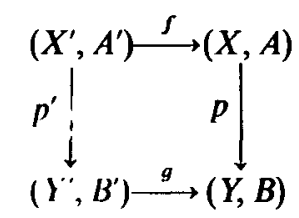


is a pull-back, then

$$
p_{u}^{\prime} f^{*}(u)=g^{*} p_{\cup}(u) \quad \text { for } \quad u \in \mathscr{H}^{*}(X, A ; M)
$$

(we identify $p_{\otimes}^{\prime} f^{*} M$ and $g^{*} p_{\otimes} M$ by 2.8 ).

3.3. If $p: X \rightarrow Y$ is the identity, then

$$
p_{\cup}(u)=u \quad \text { for } \quad u \in \mathscr{H}^{*}(X, A ; M)
$$

(note that $p_{\otimes} M=M$ by 2.9 ).

3.4. If $q:(Y, B) \rightarrow(Z, C)$ is also a fibration with finite fibres, then

$$
q_{\cup} p_{\cup}(u)=(q p)_{\cup}(u) \quad \text { for } \quad u \in \mathscr{H}^{*}(X, A ; M)
$$

(we identify $q_{\otimes} p_{\otimes} M$ and $(q p)_{\otimes} M$ by 2.10 ).

3.5. If $X$ is empty, $M$ is the unique graded local system on $X$ (so that $\mathscr{H}^{*}\left(Y, B ; p_{\otimes} M\right)=H^{0}(Y ; \mathbb{Z})$ by 2.11$)$, and 0 is the unique element of $\mathscr{H}^{*}(X, A ; M)$, then

$$
p_{\cup}(0)=1 \in H^{0}(Y ; \mathbb{Z})
$$

3.6. If $X$ is a disjoint union $X_{1} \sqcup X_{2}$ with $A_{1}=X_{1} \cap A, A_{2}=X_{2} \cap A$, and with inclusions

$$
i_{1}:\left(X_{1}, A_{1}\right) \rightarrow(X, A), \quad i_{2}:\left(X_{2}, A_{2}\right) \rightarrow(X, A)
$$

then

$$
p_{\cup}(u)=\left(p i_{1}\right)_{\cup} i_{1}^{*}(u) \cup\left(p i_{2}\right)_{\cup} i_{2}^{*}(u) \quad \text { for } \quad u \in \mathscr{H}^{*}(X, A ; M)
$$

(we identify $p_{\otimes} M$ and $\left(p i_{1}\right)_{\otimes} i_{1}^{*} M \otimes\left(p i_{2}\right)_{\otimes} i_{2}^{*} M$ by 2.12 ).

3.7. If $N$ is also a graded local system on $X$, then

$$
p_{\cup}(u \cup v)=p_{\cup}(u) \cup p_{\cup}(v) \quad \text { for } \quad u \in \mathscr{H}^{*}(X, A ; M), \quad v \in \mathscr{H}^{*}:(X, A ; N)
$$

(we identify $p_{\otimes}(M \otimes N)$ and $p_{\otimes} M \otimes p_{\otimes} N$ by 2.13).

\section{The construction of the transfer}

We begin with some remarks on Eilenberg-MacLane spaces, and then recall that $\mathscr{H}^{*}(X, A ; M)$ (and similarly $\mathscr{H}^{*}\left(Y, B ; p_{\otimes} M\right)$ ) can be regarded as a set of homotopy classes. Next we construct something analogous to an operad action (see May [8], 1), and finally construct the transfer on the lines of $[1], 4.2$. 
Let $L$ be an abelian group and $q$ be a non-negative integer. We need a definite Eilenberg-MacLane space $K(L, q)$, not just a homotopy type. In order to construct the operad action it is convenient to take $K(L, q)$ as the functional topological abelian group described by Segal in [10] (it is there denoted $S^{q} \otimes L$ ). This $K(L, q)$ is the $q$ th delooping of $L$ by Segal's infinite loop space machine [9]. One can show that these spaces $K(L, q)$ are the geometric realisations of the standard simplicial abelian groups giving Eilenberg-MacLane spaces (compare [9] with May [7], 23, for instance). The following proposition is a standard fact about these simplicial abelian groups (see [7], 23); it may also be obtained by inspection of [9].

Proposition 4.1. The space $K(L, q)$ is the geometric realisation of a functorial simplicial abelian group whose non-degenerate simplexes in degrees up to q are $\eta$, the base-point, in degree 0 and $L \backslash\{0\}$ in degree $q$.

Now let $G$ be a graded abelian group. We set

$$
K(G)=\prod_{q=0}^{\infty} K\left(G_{q}, q\right)
$$

The graded local system $M$ gives a composite functor $K M$ from $X$ to spaces, hence a bundle

$$
\bigcup_{X} K M=\bigcup_{x \in X} K M(x)
$$

over $X$. Following Siegel [11], 3.6, which gives the absolute case, we have

Proposition 4.2. The cohomology set $\mathscr{H}^{*}(X, A ; M)$ may be identified with the set of homotopy classes of sections of

$$
\bigcup_{X} K M \rightarrow X
$$

sending each point a of $A$ to a point of the form

$$
(k, 0,0, \ldots) \in K M(a)=\prod_{q=0}^{\infty} K\left(M(a)_{y}, q\right)
$$

The proof is by obstruction theory; for instance one can apply Whitehead [15], VI (6.16).

Next we recall from [8], 1 that an operad $\mathscr{C}$ is a sequence of spaces $\mathscr{C}(0), \mathscr{C}(1), \ldots$, with structure maps

$$
\gamma: \mathscr{C}(n) \times \mathscr{C}\left(m_{1}\right) \times \ldots \times \mathscr{C}\left(m_{n}\right) \rightarrow \mathscr{C}\left(m_{1}+\ldots+m_{n}\right)
$$

an identity element $1 \in \mathscr{C}(1)$, and an action of the symmetric group $\Sigma_{n}$ on $\mathscr{C}(n)$ for each $n$, satisfying various conditions. The following result gives us an "external" analogue of an operad action on the spaces $K(G)$, where $G$ runs through graded abelian groups. 
Theorem 4.3. There is an operad $\mathscr{C}$ and there are maps

$$
\theta: \mathscr{C}(n) \times K(G(1)) \times \ldots \times K(G(n)) \rightarrow K(G(1) \otimes \ldots \otimes G(n))
$$

for $G(1), \ldots, G(n)$ graded abelian groups such that

(i) $\theta$ is natural in $G(1), \ldots, G(n)$;

(ii) each space $\mathscr{C}(n)$ is contractible;

(iii) for each fixed $c \in \mathscr{C}(n)$ the map

$$
\theta(c ;-): K(G(1)) \times \ldots \times K(G(n)) \rightarrow K(G(1) \otimes \ldots \otimes G(n))
$$

is graded multilinear and represents the cup-product;

(iv) $\theta\left(\gamma\left(c ; d_{1}, \ldots, d_{n}\right) ; g(1,1), \ldots, g\left(1, m_{1}\right), \ldots, g(n, 1), \ldots, g\left(n, m_{n}\right)\right)$

$$
=\theta\left(c ; \theta\left(d_{1} ; g(1,1), \ldots, g\left(1, m_{1}\right)\right), \ldots, \theta\left(d_{n} ; g(n, 1), \ldots, g\left(n, m_{n}\right)\right)\right)
$$

for $c \in \mathscr{C}(n), d_{i} \in \mathscr{C}\left(m_{i}\right), g(i, j) \in K(G(i, j))$;

(v) $\theta(1 ; g)=g$ for $1 \in \mathscr{C}(1)$ the identity and $g \in K(G)$;

(vi) $\theta(c \cdot \sigma ; g(\sigma 1), \ldots, g(\sigma n)) \cdot \sigma=\theta(c ; g(1), \ldots, g(n))$ for $c \in \mathscr{C}(n), \sigma \in \Sigma_{n}, g(i) \in K(G(i))($ where

$$
\sigma: K(G(\sigma 1) \otimes \ldots \otimes G(\sigma n)) \rightarrow \dot{K}(G(1) \otimes \ldots \otimes G(n))
$$

is given by permuting factors and putting in signs according to the anticommutative convention).

Proof. This is almost the same as in [14], 2. For $q=(q(1), \ldots, q(n))$ an $n$-tuple of nonnegative integers with $q(1)+\ldots+q(n)=q$ we have a contractible space $\mathscr{C}(\mathbf{q})$ consisting of the natural multilinear maps

$$
K(L(1), q(1)) \times \ldots \times K(L(n), q(n)) \rightarrow K(L(1) \otimes \ldots \otimes L(n), q)
$$

representing the cup-product $(L(1), \ldots, L(n)$ run through abelian groups). We set

$$
\mathscr{C}(n)=\prod_{\mathbf{q}} \mathscr{C}(\mathbf{q})
$$

the product being taken over all $n$-tuples q. One can check that the $\mathscr{C}(n)$ with the obvious structure form an operad $\mathscr{C}$, and (ii) follows from the contractibility of the $\mathscr{C}(\mathbf{q})$.

To construct the maps $\theta$, let $c=\left(c_{q}\right) \in \mathscr{C}(n)$ and

$$
g(i)=\left(g(i)_{q(i)}\right) \in K(G(i))=\prod_{q(i)=0}^{\infty} K\left(G(i)_{q(i)}, q(i)\right)
$$


Write $\theta(c ; g(1), \ldots, g(n))$ in the form

$$
\left(h_{q}\right) \in K(G(1) \otimes \ldots \otimes G(n))=\prod_{q=0}^{\infty} K\left((G(1) \otimes \ldots \otimes G(n))_{q}, q\right) .
$$

For $q$ an $n$-tuple with $q(1)+\ldots+q(n)=q$ let

$$
\iota_{\mathrm{q}}: K\left(G(1)_{q(1)} \otimes \ldots \otimes G(n)_{q(n)}, q\right) \rightarrow K\left((G(1) \otimes \ldots \otimes G(n))_{q}, q\right)
$$

be induced by the obvious inclusion. Then $h_{q}$ is to be

$$
\sum_{q} l_{q} c_{q}\left(g(1)_{q(1)}, \ldots, g(n)_{q(n)}\right)
$$

where the sum is taken over $n$-tubles q such that $q(1)+\ldots+q(n)=q$.

One can now check (i) and (iii)-(vi). This completes the proof.

We now construct the transfer from 4.3 by the method of [1], 4.2, applying it to sections of bundles rather than maps into fixed spaces. Recall that we have a fibration $p: X \rightarrow Y$ with finite fibres and functors $K M$ and $K\left(p_{\otimes} M\right)$ from $\pi X$ and $\pi Y$ respectively to spaces. According to 4.2 the transfer has to map certain homotopy classes of sections of $\bigcup_{X} K M$ to homotopy classes of sections of $\bigcup_{Y} K\left(p_{\otimes} M\right)$.

Let us write $\Pi X$ for $\sqcup_{n=0}^{\infty} X^{n}$. We have functors $\mathscr{C}$ and $\Pi K M$ from $\pi \Pi X$ to spaces given by:

$$
\mathscr{C}\left(x_{1}, \ldots, x_{n}\right)=\mathscr{C}(n) \text { and } \Pi K M\left(x_{1}, \ldots, x_{n}\right)=K M\left(x_{1}\right) \times \ldots \times K M\left(x_{n}\right)
$$

for $\left(x_{1}, \ldots, x_{n}\right) \in X^{n} ; \mathscr{C}$ sends morphisms of $\pi \Pi X$ to identity maps; and $\Pi K M$ acts on morphisms in the obvious way. These functors give us a product functor $\mathscr{C} \times \Pi K M$ from $\pi \Pi X$ to spaces with

$$
(\mathscr{C} \times \Pi K M)(x)=\mathscr{C}(x) \times \Pi K M(x) \quad \text { for } \quad x \in \Pi X
$$

We also have a graded local system $\bigotimes M$ on $\Pi X$ given by

$$
(\otimes M)\left(x_{1}, \ldots, x_{n}\right)=M\left(x_{1}\right) \otimes \ldots \otimes M\left(x_{n}\right)
$$

for $\left(x_{1}, \ldots, x_{n}\right) \in X^{n}$. This gives us a functor $K(\bigotimes M)$ from $\pi \Pi X$ to spaces. We can now see that 4.3 gives us a natural transformation

$$
\theta: \mathscr{C} \times \Pi K M \rightarrow K(\bigotimes M)
$$

Next we define a subspace $p_{*} X$ of $\Pi X$ by the condition that $\left(x_{1}, \ldots, x_{n}\right)$ is in $p_{*} X$ if and only if $x_{1}, \ldots, x_{n}$ are distinct points making up a fibre of $p: X \rightarrow Y$. One might say that $p_{*} X$ is the space of fibres of $p$. We note that the part of $p_{*} X$ lying in $X^{n}$ is a 
principal $\Sigma_{n}$-bundle, and that $Y$ is the disjoint union of the orbit spaces. We shall express this by saying that $\Sigma$ acts freely on $p_{*} X$ and

$$
\left(p_{*} X\right) / \Sigma=Y \text {. }
$$

Since $\mathscr{C}(n)$ is a contractible space (4.3(ii)) on which $\Sigma_{n}$ acts, there is an equivariant map from $p_{*} X \cap X^{n}$ to $\mathscr{C}(n)$ determined up to equivariant homotopy. These maps fit together to give an equivariant section

$$
\lambda: p_{*} X \rightarrow \bigcup_{p * x} \mathscr{C}
$$

determined up to equivariant homotopy.

Now let $z: X \rightarrow \bigcup_{X} K M$ be a section representing a class $u$ in $\mathscr{H}^{*}(X, A ; M)$ (see 4.2). In the obvious way it gives us a section

$$
\Pi z: \Pi X \rightarrow \bigcup_{\Pi X} \Pi K M .
$$

From 4.4-4.6 we obtain a composite section

$$
p_{*} X \stackrel{(2, \Pi z)}{\longrightarrow} \bigcup_{p_{*} X}(\mathscr{C} \times \Pi K M) \stackrel{\cup_{\theta}}{\longrightarrow} \bigcup_{p_{0} X} K(\otimes M)
$$

One can check that $(\bigcup \theta) \circ(\lambda, \Pi z)$ is equivariant, so yields a section

$$
w: Y=\left(p_{*} X\right) / \Sigma \rightarrow\left(\bigcup_{p_{*} X} K(\otimes M)\right) / \Sigma=\bigcup_{Y} K\left(p_{\otimes} M\right) .
$$

Using the multilinearity of $\theta$ (4.3(iii)) one can check that $w$ represents a class in $\mathscr{H}^{*}\left(Y, B ; p_{\otimes} M\right)$. Since $\lambda$ is determined up to equivariant homotopy, the class represented by $w$ depends only on the equivariant homotopy class of $\Pi z$; that is, it depends only on the homotopy class of $z$. So the class $u$ in $\mathscr{H}^{*}(X, A ; M)$ represented by $z$ gives us a class $v$ in $\mathscr{H}^{*}\left(Y, B ; p_{\otimes} M\right)$ represented by $w$, and $v$ depénds only on $u$. We define $p_{\cup}(u)$ to be $v$.

\section{Proofs of the formal properties}

We now prove the properties 3.1-3.7 for the transfer constructed in Section 4.

Proof of 3.1. This follows from the naturality of $\theta$ (4.3(i)).

Proof of 3.2. Suppose that we use a section $\lambda: p_{*} X \rightarrow \bigcup_{p * X} \mathscr{C}$ in the construction of $p_{\cup}$. If $x^{\prime} \in p_{*}^{\prime} X^{\prime}$, then $\mathscr{C}\left(x^{\prime}\right)=\mathscr{C}\left((\Pi f)\left(x^{\prime}\right)\right)$, and we can define an equivariant section $\lambda^{\prime}: p_{*}^{\prime} X^{\prime} \rightarrow \bigcup_{p: X^{\prime}} \mathscr{C}$ by

$$
\lambda^{\prime}\left(x^{\prime}\right)=\lambda\left((\Pi f)\left(x^{\prime}\right)\right) \quad \text { for } \quad x^{\prime} \in p_{*}^{\prime} X^{\prime}
$$


If we use the sections $\lambda$ and $\lambda^{\prime}$ in the construction of $p_{v}$ and $p_{u}^{\prime}$, then 3.2 is true at the level of sections.

Proof of 3.3. Here $p_{*} X=X=Y, \mathscr{C}(x)=\mathscr{C}(1)$ for $x \in p_{*} X$, and we can define an equivariant section $\lambda: p_{*} X \rightarrow \bigcup_{p_{*} X} \mathscr{C}$ by

$$
\lambda(x)=1 \in \mathscr{C}(1) \quad \text { for } \quad x \in p_{*} X .
$$

If we use this $\lambda$ in the construction of $p_{v}$, then 3.3 is true at section-level by $4.3(v)$.

Proof of 3.4. Let $z$ be a section representing $u$. Suppose we use equivariant sections

$$
\lambda: p_{*} X \rightarrow \bigcup_{p_{*} X} \mathscr{C} \text { and } \mu: q_{*} Y \rightarrow \bigcup_{q_{*} Y} \mathscr{C}
$$

to construct $p_{\cup}$ and $q_{u}$. Using the equivariance properties of $\gamma$, the structure map of the operad $\mathscr{C}$ (see [8], 1.1(c) for these), we find that there is an equivariant section

$$
v:(q p)_{*} X \rightarrow \bigcup_{(q p)_{*} X} \mathscr{C}
$$

making the square

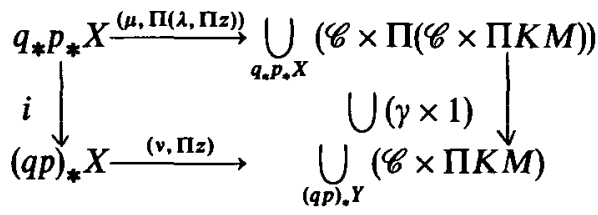

equivariantly commutative, where $i$ is the obvious inclusion. On the other hand 4.3(iv) shows that the square

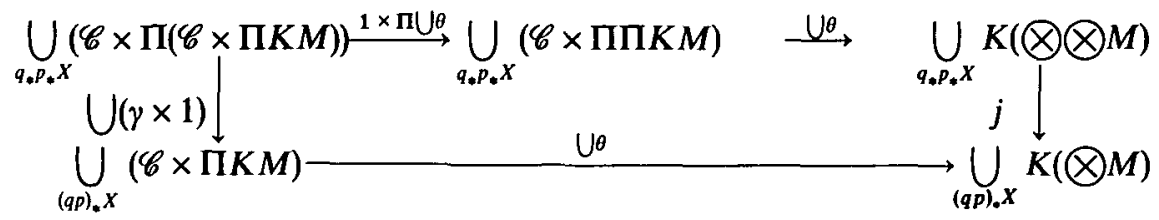

is equivariantly commutative, where $j$ is the obvious inclusion. Now a section $s$ representing $q_{\cup} p_{\cup}(u)$ is obtained from

$$
(\bigcup \theta) \circ(1 \times \Pi \bigcup \theta) \circ(\mu, \Pi(\lambda, \Pi z))
$$

by passing to orbit spaces twice, while a section $t$ representing $(q p)_{\cup}(u)$ is obtained from

$$
(\bigcup \theta) \circ(v, \Pi z)
$$


by passing to orbit spaces once. It follows from the commutativity of the two squares that $s=t$, so $q_{\cup} p_{\cup}(u)=(q p)_{\cup}(u)$ as required.

Proof of 3.5. This follows from the conventions (see 2.6).

Proof of 3.6. By 3.2-3.4 it suffices to consider the case $X_{1}=X_{2}=Y, A_{1}=A_{2}=B$, and $p: X=Y \sqcup Y \rightarrow Y$ is the standard collapsing map. We then have to show that

$$
p_{\cup}(u)=i_{i}^{*}(u) \cup i_{2}^{*}(u) \quad \text { for } \quad u \in \mathscr{H}^{*}(X, A ; M) \text {. }
$$

Now $p_{*} X$ is the disjoint union of two copies of the diagonal of $Y^{2}$, which are interchanged by the non-trivial element $\tau$ of $\Sigma_{2}$. An equivariant section $\lambda: p_{*} X \rightarrow \bigcup_{p_{*} X} \mathscr{C}$ can be obtained by sending one copy to a fixed element $c$ of $\mathscr{C}(2)$ (note that every fibre is $\mathscr{C}(2))$ and the other copy to $c \cdot \tau$. If we use this $\lambda$ to construct $p_{v}$, then the result follows from $4.3($ iii).

Proof of 3.7. This follows from 3.3, 3.4 and 3.6 by considering the composite fibration

$$
(X \sqcup X, A \sqcup A) \stackrel{\text { collapse }}{\longrightarrow}(X, A) \stackrel{p}{\longrightarrow}(Y, B) .
$$

\section{The transfer in terms of cochains}

In this section we describe the transfer

$$
p_{\cup}: \mathscr{H}^{*}(X, A ; M) \rightarrow \mathscr{H}^{*}\left(Y, B ; p_{\otimes} M\right)
$$

in terms of cochains. We suppose for simplicity that the fibres of $p$ have constant cardinality $n$; the general case reduces to this by splitting $Y$ into components. We shall write $S(Z)$ for the singular complex of a space $Z$; and if $N$ is a graded local system on $Z$, then we shall write $\bigoplus_{z} G$ for $\bigoplus_{z \in Z} G(z)$.

Suppose that $u \in \mathscr{H}^{*}(X, A ; M)$ is represented by a cocycle

$$
c: S(X) \rightarrow \bigoplus_{X} M
$$

Then $c$ will be a graded homomorphism over $X$; that is, if $\sigma: \Delta^{q} \rightarrow X$ is a singular $q$ simplex with leading vertex $\sigma(0) \in X\left(0\right.$ here denotes the leading vertex of $\left.\Delta^{q}\right)$, then

$$
c(\sigma) \in M(\sigma(0))_{q}
$$

We have to find a cocycle

over $Y$ representing

$$
c^{\prime}: S(Y) \rightarrow \bigoplus_{Y}\left(p_{\otimes} M\right)
$$

$$
p_{\cup}(u) \in \mathscr{H}^{*}\left(Y, B ; p_{\otimes} M\right)
$$


To do this we use ideas based on acyclic models; see Spanier [12], 4.2 and Dold [3], 1. Let $W$ be a resolution of $\mathbb{Z}$ by free $\mathbb{Z} \Sigma_{n}$-modules; by [3], 1.12 we have a natural equivariant chain homotopy equivalence

$$
\alpha: W \otimes S\left(X^{n}\right) \rightarrow \stackrel{\bigotimes}{\bigotimes}^{\prime} S(X)
$$

from $\alpha$ and

$$
\bigotimes^{n} c: \bigotimes^{n} S(X) \rightarrow \bigotimes^{n}\left(\bigoplus_{X} M\right)=\bigoplus_{X^{n}}(\otimes M)
$$

$(\otimes M$ is defined in Section 4$)$ we construct an equivariant cocycle

$$
c^{n}=c \times \ldots \times c: W \otimes S\left(X^{n}\right) \rightarrow \bigoplus_{X^{n}}(\otimes M)
$$

over $X^{n}$ (see Remark 6.2 below). By our assumption on the cardinality of the fibres of $p$ we have an inclusion map $i: p_{*} X \rightarrow X^{n}$; we therefore have an equivariant cocycle

$$
i^{*}\left(c^{n}\right)=c^{n} \circ(1 \times i): W \otimes S\left(p_{*} X\right) \rightarrow \bigoplus_{p_{*} X}(\bigotimes M)
$$

over $p_{*} X$. The augmentation $\varepsilon: W \rightarrow \mathbb{Z}$ induces an equivariant homology equivalence

$$
\varepsilon \otimes 1: W \otimes S\left(p_{*} X\right) \rightarrow S\left(p_{*} X\right),
$$

and $\varepsilon \otimes 1$ has an equivariant chain homotopy inverse

$$
\beta: S\left(p_{*} X\right) \rightarrow W \otimes S\left(p_{*} X\right)
$$

because $\Sigma_{n}$ acts freely on $p_{*} X$ (so that the complexes consist of free $\mathbb{Z} \Sigma_{n}$-modules). Acyclic model arguments show that the equivariant chain homotopy class of

$$
\alpha \circ(1 \otimes i) \circ \beta: S\left(p_{*} X\right) \rightarrow \bigotimes^{n} S(X)
$$

is independent of the choices of $W, \alpha$ and $\beta$.

Putting all this together, we obtain an equivariant cocycle

$$
i^{*}\left(c^{n}\right) \circ \beta: S\left(p_{*} X\right) \rightarrow \underset{p_{0} X}{\bigoplus}(\bigotimes M)
$$

over $p_{*} X$ whose equivariant cohomology class is independent of any choices. By passing to orbits we obtain a cocycle

$$
c^{\prime}: S(Y)=S\left(p_{*} X\right) / \Sigma_{n} \rightarrow\left(\bigoplus_{p_{*} X}(\otimes M)\right) / \Sigma_{n}=\bigoplus_{Y}\left(p_{\otimes} M\right)
$$

whose cohomology class is independent of any choices. 
We shall prove the following result.

Theorem 6.1. The cocycle $c^{\prime}$ has cohomology class $p_{\cup}(u)$ in $\mathscr{H}^{*}\left(Y, B ; p_{\otimes} M\right)$.

Remark 6.2. The definition of $c^{n}$ requires a little care. It is essentially the composite

$$
\bigotimes^{n} c \circ \alpha: W \otimes S\left(X^{n}\right) \rightarrow \bigoplus_{X^{n}}(\otimes M)
$$

but we have to make it lie over $X^{n}$ by using the morphisms of $\pi X$. To do this let $w \in W$ and $\sigma: \Delta^{q} \rightarrow X^{n}$, and suppose that $\sigma$ has components $\sigma_{1}, \ldots, \sigma_{n}: \Delta^{q} \rightarrow X$. By the naturality of $\alpha$ we shall have

$$
\alpha(w \otimes \sigma)=\sum_{k} \sigma_{1} \tau_{1 k} \otimes \ldots \otimes \sigma_{n} \tau_{n k}
$$

for certain singular simplexes $\tau_{i k}$ in $\Delta^{q}$. We define $c^{n}(w \otimes \sigma)$ to be

$$
\sum_{k} \omega_{1 k^{*}} c\left(\sigma_{1} \tau_{1 k}\right) \otimes \ldots \otimes \omega_{n k^{*}} c\left(\sigma_{n} \tau_{n k}\right)
$$

where $\omega_{i k}: \sigma_{i} \tau_{i k}(0) \rightarrow \sigma_{i}(0)$ in $\pi X$ is the image under $\sigma_{i^{*}}$ of the unique morphism from $\tau_{i k}(0)$ to 0 in $\Delta^{q}$. Compare the definition of the cup-product of local cochains in [13], 11. We shall say that $c^{n}$ is $\left.\otimes\right)^{n} c \circ \alpha$ made vertical.

To prove Theorem 6.1 we proceed as follows. Let $z: X \rightarrow \bigcup_{X} K M$ be a section representing $u$. Then $p_{u}(u)$ is represented by the section $w$ of 4.8 , which is covered by the equivariant section $(\bigcup \theta) \circ(\lambda, \Pi z)$ of 4.7 . Let

$$
\bigcup \imath: S\left(\bigcup_{p_{\bullet} X} K(\bigotimes M)\right) \rightarrow \bigoplus_{p_{0} X}(\otimes M)
$$

be an equivariant classifying cocycle. We have

$$
(\bigcup \imath)(\sigma)=\imath(|\sigma|)
$$

for $\sigma$ a singular simplex in $\bigcup_{p . X} K(\otimes M),|\sigma|$ the projection of $\sigma$ onto the fibre through its leading vertex, and $\imath$ a classifying cocycle of this fibre. We can make $\bigcup_{l}$ equivariant, because $\Sigma_{n}$ acts freely on $\bigcup_{p * x} K(\otimes M)$. The basic constructions of obstruction theory (see for instance [15], VI.5-6) now show that $p_{\cup}(u)$ is the class of the cocycle covered by the equivariant cocycle

$$
S\left(p_{*} X\right) \stackrel{(\lambda, \Pi z)}{\longrightarrow} S\left(\bigcup_{p_{*} X}(\mathscr{C} \times \Pi K M)\right) \stackrel{U^{\theta}}{\longrightarrow} S\left(\bigcup_{p_{*} X} K(\bigotimes M)\right) \stackrel{U_{i}}{\longrightarrow} \bigoplus_{p_{*} X}(\bigotimes M)
$$


So we have to show that this cocycle is equivariantly cohomologous to the cocycle $i^{*}\left(c^{n}\right) \circ \beta$ covering the cocycle $c^{\prime}$ of 6.1 .

To do this, we shall replace the complexes in 6.3 by chain homotopy equivalent complexes in such a way that the problem reduces to considering $\theta$ fibre by fibre. Suppose in general that $Z$ is a space and $F$ a functor from $\pi Z$ to spaces. For fixed $q \geqq 0$ we have a functor $S_{q}(F)$ from $\pi Z$ to abelian groups. Let $S\left(Z ; S_{q}(F)\right.$ ) be the chain complex whose homology is $H_{*}\left(Z ; S_{q}(F)\right)$; as a graded abelian group we have

$$
S\left(Z ; S_{q}(F)\right)=\bigoplus_{z \in Z} S(Z, z) \otimes S_{q}(F(z))
$$

where $S(Z, z)$ is the subgroup of $S(Z)$ generated by simplexes with leading vertex $z$. On varying $q$ we obtain a double complex $S(Z ; S(F))$.

Theorem 6.4. There is a natural chain homotopy equivalence

$$
S(Z ; S(F)) \simeq S\left(\bigcup_{Z} F\right)
$$

This is proved by acyclic models, the models being the constant functors $F$ on $\pi \Delta^{i}$ with $F(z)=\Delta^{j}$ for $z \in \Delta^{i}$. The proof and the result are similar to those of Brown [2], but easier.

We note that 6.4 easily yields Serre spectral sequences. Also, if $F$ is a constant functor $\left(F(z)=Z^{\prime}\right.$ for $\left.z \in Z\right)$, then 6.4 becomes the equivalence

$$
S(Z) \otimes S\left(Z^{\prime}\right) \simeq S\left(Z \times Z^{\prime}\right)
$$

of Eilenberg and Zilber [4].

We return to considering 6.3. Since every fibre of $p$ has cardinality $n$,

$$
\bigcup_{p_{*} X}(\mathscr{C} \times \Pi K M)=\mathscr{C}(n) \times \bigcup_{p_{*} X} \Pi K M
$$

Let $V$ be a resolution of $\mathbb{Z}$ by free $\mathbb{Z} \Sigma_{n}$-modules. Since $\mathscr{C}(n)$ is contractible (4.3(ii)), $W$ $=V \otimes S(\mathscr{C}(n))$ is also a resolution of $\mathbb{Z}$ by free $\mathbb{Z} \Sigma_{n}$-modules. We now see that under the equivalence $6.5(\lambda, \Pi z)$ is equivariantly equivalent to the composite

$$
S\left(p_{*} X\right) \stackrel{\beta}{\longrightarrow} W \otimes S\left(p_{*} X\right)=V \otimes S(\mathscr{C}(n)) \otimes S\left(p_{*} X\right) \stackrel{\leftarrow \otimes 1 \otimes n}{\longrightarrow} S(\mathscr{C}(n)) \otimes S\left(\bigcup_{p_{*} X} \Pi K M\right)
$$

where $\beta$ is an equivariant chain homotopy inverse to $\varepsilon \otimes 1$. Using 6.4 and 6.5 we then see that the cocycle of 6.3 is equivariantly cohomologous to 


$$
\begin{aligned}
& S\left(p_{*} X\right) \stackrel{\beta}{\longrightarrow} W \otimes S\left(p_{*} X\right)=V \otimes S(\mathscr{C}(n)) \otimes S\left(p_{*} X\right) \\
& \stackrel{\bullet \otimes 1 \otimes \Pi z}{\longrightarrow} S(\mathscr{C}(n)) \otimes S\left(\bigcup_{p_{0} X} \Pi K M\right) \\
& \simeq S(\mathscr{C}(n)) \otimes S\left(p_{*} X ; S(\Pi K M)\right)=S\left(p_{*} X ; S(\mathscr{C}(n)) \otimes S(\Pi K M)\right) \\
& \simeq S\left(p_{*} X ; S(\mathscr{C}(n) \times \Pi K M)\right) \\
& \stackrel{1 \otimes \theta}{\longrightarrow} S\left(p_{*} X ; S(K(\bigotimes M))\right) \\
& \stackrel{\varepsilon \otimes 1}{\longrightarrow} \bigoplus_{p_{*} X}(\otimes M)
\end{aligned}
$$

made vertical: the morphisms of $p_{*} X$ act trivially on $S(\mathscr{C}(n))$, and the last mosphism is $\varepsilon \otimes \imath$ because $\bigcup_{l}$ is projection onto the fibre followed by $l$.

A comparison of this with the cocycle of 6.1 shows that the proof of 6.1 reduces to the following result.

Proposition 6.6. If $G(1), \ldots, G(n)$ are graded abelian groups, then the composite

$$
\begin{gathered}
S(\mathscr{C}(n)) \otimes S(K G(1) \times \ldots \times K G(n)) \simeq S(\mathscr{C}(n) \times K G(1) \times \ldots \times K G(n)) \\
\stackrel{\theta}{\longrightarrow} S(K(G(1) \otimes \ldots \otimes G(n))) \stackrel{!}{\longrightarrow} G(1) \otimes \ldots \otimes G(n)
\end{gathered}
$$

is cohomologous to $\varepsilon \otimes \mu$, where

$$
\mu: S(K G(1) \times \ldots K G(n)) \rightarrow G(1) \otimes \ldots \otimes G(n)
$$

represents the cross-product of the universal classes.

Proof. For $L$ an abelian group and $q \geqq 0$, let $C(L, q)$ be the simplicial chain complex of $K(L, q)(4.1$ tells us that $K(L, q)$ is the geometric realisation of a simplicial set). Then the inclusion of $C(L, q)$ in $S(K(L, q))$ is a homology equivalence ([12], 4.6.11). Combining this with 6.5 gives a natural homology equivalence

$$
\gamma: S(\mathscr{C}(n)) \otimes \bigotimes_{i=1}^{n} \bigotimes_{q=0}^{\infty} C\left(G(i)_{q}, q\right) \rightarrow S(\mathscr{C}(n) \times K G(1) \times \ldots \times K G(n))
$$

(recall that $K G(i)$ is

$$
\left.\prod_{q=0}^{\infty} K\left(G(i)_{q}, q\right)\right)
$$


It follows from 4.1 that $C\left(G(i)_{q}, q\right)$ has a basis

$$
B_{i q}=\{\eta\} \cup\left(G(i)_{q} \backslash\{0\}\right) \cup B_{i q}^{\prime}
$$

with $\operatorname{deg}(\eta)=0, \operatorname{deg}(g)=q$ for $g \in G(i)_{q} \backslash\{0\}, \operatorname{deg}(b)>q$ for $b \in B_{i q}^{\prime}$. A typical basis element for the domain of $\gamma$ therefore has the form

$$
\tau=\sigma \otimes \bigotimes_{i=1}^{n} \bigotimes_{q=0}^{\infty} b_{i q}
$$

with $\sigma$ a singular simplex in $\mathscr{C}(n), b_{i q} \in B_{i q}, b_{i q}=\eta$ for all but finitely many pairs $i, q$. It now suffices to show that

(i) $i \theta \gamma(\tau)=g_{1} \otimes \ldots \otimes g_{n} \quad$ if $\quad \operatorname{deg}(\sigma)=0 \quad$ and $\quad \otimes_{q} b_{i q}=\eta \otimes \ldots \otimes \eta \otimes g_{i} \otimes \eta \otimes \ldots$ $\left(g_{i} \in G(i)_{q(i)} \backslash\{0\}\right)$ for each $i$,

(ii) $\imath \theta \gamma(\tau)=0$ otherwise.

Now (i) is true because $\theta$ represents the cup-product (4.3(iii)). And if $\tau$ is as in (ii), then we can find non-negative integers $d_{1}, \ldots, d_{n}$ such that $b_{i q}=\eta$ for $q>d_{i}$ and $d_{1}+\ldots$ $+d_{n}<\operatorname{deg}(\tau)$. Let $H(i)$ be the graded subgroup of $G(i)$ obtained by truncation at $d_{i}$; that is,

$$
\begin{aligned}
& H(i)_{q}=G(i)_{q} \text { for } q \leqq d_{i} \\
& 0 \text { for } q>d_{i} \text {. }
\end{aligned}
$$

Then the naturality of $\theta$ (4.3(i)) shows that $\imath \theta \gamma(\tau)$ lies in

$$
\operatorname{Im}[H(1) \otimes \ldots \otimes H(n) \rightarrow G(1) \otimes \ldots \otimes G(n)] .
$$

But this vanishes in the degree of $\tau$, since $d_{1}+\ldots+d_{n}<\operatorname{deg}(\tau)$, so $\imath \theta \gamma(\tau)=0$, as required.

This completes the proof of (ii), hence of 6.6, hence of 6.1 .

\section{The double coset rule}

Suppose that $G, H$ and $K$ are groups, $i: H \rightarrow G$ is the inclusion of a subgroup of finite index, and $f: K \rightarrow G$ is a homomorphism. We then have the transfer

$$
i_{\cup}: H^{*}(H ; M) \rightarrow H^{*}\left(G ; i_{\otimes} M\right)
$$

for $M$ a graded $H$-module. The double coset rule describes the composite

$$
f^{*} i_{u}: H^{*}(H ; M) \rightarrow H^{*}\left(K ; f^{*} i_{\otimes} M\right)
$$

(Often $f$ is required to be an inclusion too, but this is not necessary.) 
To state the rule, write $G$ as a disjoint union of double cosets

$$
G=\bigsqcup_{r=1}^{d} f(K) g_{r} H
$$

Let $K_{r}$ be $f^{-1}\left(g_{r} H g_{r}^{-1}\right), j_{r}: K_{r} \rightarrow K$ be the inclusion, and $c_{r}: K_{r} \rightarrow H$ be given by

$$
c_{r}(k)=g_{r}^{-1} f(k) g_{r} \quad \text { for } \quad k \in K_{r} .
$$

The rule then says that

$$
f^{*} i_{\otimes} M \cong \bigotimes_{r=1}^{d} j_{r \otimes} c_{r}^{*} M
$$

and that with this identification

$$
f^{*} i_{\cup}(u)=\prod_{r=1}^{d} j_{r u} c_{r}^{*}(u) \quad \text { for } \quad u \in H^{*}(H ; M)
$$

The rule is proved by applying $2.8,2.12,3.2$ and 3.6 to the following geometric result.

Lemma 7.3. The square

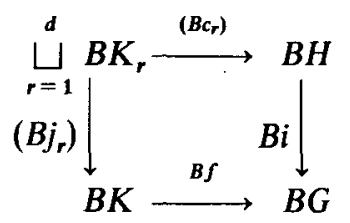

is homotopy equivalent to a pull-back square whose columns are fibrations with finite fibres.

The proof of 7.3 is straightforward, and will be omitted.

\section{REFERENCES}

1. J. F. Adams, Infinite Loop Spaces (Annals of Mathematics Studies 90, Princeton University Press and University of Tokyo Press, Princeton, 1978).

2. E. H. Brown, Twisted tensor products, I, Ann. Math. 69 (1959), 223-246.

3. A. Dold, Über die steenrodschen Kohomologieoperationen, Ann. Math. 73 (1961), 258-294.

4. S. Eilenberg and J. A. Zilber, On products of complexes, Amer. J. Math. 75 (1953), 200-204.

5. L. EvENS, A generalization of the transfer map in the cohomology of groups, Trans. Amer. Math. Soc. 108 (1963), 54-65.

6. D. S. Kahn and S. B. Priddy, The transfer and stable homotopy theory, Math. Proc. Cambridge Philos. Soc. 83 (1978), 103-111. 
7. J. P. MAY, Simplicial Objects in Algebraic Topology (Van Nostrand Mathematical Studies 11 , Van Nostrand, Princeton, Toronto, London, Melbourne, 1967).

8. J. P. MaY, The Geometry of Iterated Loop Spaces (Lecture Notes in Mathematics 271, Springer, Berlin, Heidelberg, New York, 1972).

9. G. SEGAL, Categories and cohomology theories, Topology 13 (1974), 293-312.

10. G. Segal, The multiplicative group of classical cohomology, Quart. J. Math. Oxford (2) 26 (1975), 289-293.

11. J. SieGEL, Cohomology and homology theories for categories of principal G-bundles, Trans. Amer. Math. Soc. 120 (1965), 428-437.

12. E. H. Spanier, Algebraic Topology (Tata McGraw-Hill, Bombay, New Delhi, 1966).

13. N. E. Steenrod, Homology with local coefficients, Ann. Math. 44 (1943), 610-627.

14. R. SteIner, Decompositions of groups of units in ordinary cohomology, Quart. J. Math. Oxford (2) 30 (1979), 483-494.

15. G. W. WhiteheAd, Elements of Homotopy Theory (Graduate Texts in Mathematics 61, Springer, New York, Heidelberg, Berlin, 1978).

University of Glasgow

Department of Mathematics

15 University GaRdens

Glasgow G12 8QW 